ARTICLE

\title{
FAM21 directs SNX27-retromer cargoes to the plasma membrane by preventing transport to the Golgi apparatus
}

Seongju Lee ${ }^{1, \star}$, Jaerak Chang ${ }^{1,2,3,4, \star} \&$ Craig Blackstone ${ }^{1}$

The endosomal network maintains cellular homeostasis by sorting, recycling and degrading endocytosed cargoes. Retromer organizes the endosomal sorting pathway in conjunction with various sorting nexin (SNX) proteins. The SNX27-retromer complex has recently been identified as a major endosomal hub that regulates endosome-to-plasma membrane recycling by preventing lysosomal entry of cargoes. Here, we show that SNX27 directly interacts with FAM21, which also binds retromer, within the Wiskott-Aldrich syndrome protein and SCAR homologue (WASH) complex. This interaction is required for the precise localization of SNX27 at an endosomal subdomain as well as for recycling of SNX27-retromer cargoes. Furthermore, FAM21 prevents cargo transport to the Golgi apparatus by controlling levels of phosphatidylinositol 4-phosphate, which facilitates cargo dissociation at the Golgi. Together, our results demonstrate that the SNX27-retromer-WASH complex directs cargoes to the plasma membrane by blocking their transport to lysosomes and the Golgi.

\footnotetext{
${ }^{1}$ Cell Biology Section, Neurogenetics Branch, National Institute of Neurological Disorders and Stroke, National Institutes of Health, Bethesda, Maryland 20892, USA. ${ }^{2}$ Department of Biomedical Sciences, Ajou University School of Medicine, Suwon 443-380, Korea. ${ }^{3}$ Department of Brain Science, Ajou University School of Medicine, Suwon 443-380, Korea. ${ }^{4}$ Neuroscience Graduate Program, Ajou University School of Medicine, Suwon 443-380, Korea.

* These authors contributed equally to this work. Correspondence and requests for materials should be addressed to C.B. (email: blackstc@ninds.nih.gov).
} 
$\mathrm{T}$ he endosomal network controls numerous cellular functions such as signalling, nutrient uptake and development through balanced trafficking of diverse plasma membrane proteins ${ }^{1,2}$. After receiving membrane protein cargoes, endosomes sort them to lysosomes for degradation or else to the trans-Golgi network (TGN) or plasma membrane for recycling ${ }^{3,4}$. This endosomal sorting pathway is elaborately regulated by retromer and its interacting proteins ${ }^{5,6}$. Retromer, a multi-subunit complex that associates with the cytosolic face of endosomes, was originally identified for its ability to regulate retrograde transport from endosomes to the $\mathrm{TGN}^{7-10}$. Recent studies have expanded its regulatory role in not only endosome-to-TGN transport but also in endosome-toplasma membrane transport ${ }^{6,11}$.

Retromer comprises a core trimer of vacuolar protein sortingassociated protein 35 (VPS35), VPS26 and VPS29 as well as sorting nexin (SNX) proteins that bind phosphoinositides ${ }^{12}$. Although the composition of the VPS trimer is conserved, it forms complexes with various SNX proteins. Interactions of the VPS trimer with SNX1/2 and SNX5/6 regulate retrograde transport of cation-independent mannose-6-phosphate receptors from endosomes to the $\mathrm{TGN}^{13}$. For retrograde transport of Wntless to the TGN, retromer associates with SNX3 (ref. 14). More recently, the retromer complex associated with SNX27, a PDZ-domain-containing SNX protein, has been shown to regulate endosome-to-plasma membrane recycling of membrane proteins including the $\beta 2$-adrenergic receptor $(\beta 2 \mathrm{AR})$ and the glucose transporter GLUT1 (refs 6,11). Thus, SNX proteins appear to facilitate the cargo specificity of retromer ${ }^{15,16}$. However, mechanisms for how retromer specifies destinations of individual cargoes remain unclear.

The Wiskott-Aldrich syndrome protein and SCAR homologue (WASH) complex is a large, multiprotein complex consisting of family with sequence similarity 21 (FAM21), strumpellin, SWIP, CCDC53 and WASH1; it activates Arp2/3-mediated actin polymerization on endosomes ${ }^{17-19}$. WASH complex depletion causes endosomal sorting defects in pathways such as recycling of transferrin (Tf) receptor and integrins to the plasma membrane as well as retrograde transport of cation-independent mannose-6phosphate receptor to the Golgi apparatus $20-22$. Given that both SNX27 and retromer bind the WASH complex, the WASH complex has been suggested to function in recycling of SNX27-retromer cargoes, although a specific role has not been identified ${ }^{6,23}$

Here, we have identified an interaction of SNX27 with FAM21, the largest WASH component, which is required for proper recycling of SNX27-retromer cargoes. This interaction restricts SNX27 to an endosomal subdomain, aiding export of cargoes to their destinations. Moreover, FAM21 prevents cargoes from being transported to the Golgi apparatus by controlling Golgi levels of phosphatidylinositol 4-phosphate [PI(4)P], which facilitates a release of retromer cargoes around the Golgi.

\section{Results}

FAM21 interacts directly with SNX27. To identify the WASH complex component that binds SNX27 directly, we conducted in vitro pull-down assays. Among the five core proteins of the WASH complex, only FAM21 interacted with SNX27 fusion proteins purified from bacteria (Fig. 1a). FAM21 contains an $\mathrm{N}$-terminal globular head domain and a C-terminal unstructured tail harbouring multiple L-F-[D/E $]_{3-10}-\mathrm{L}-\mathrm{F}$ (LFa) motifs (Fig. 1b); it regulates the WASH complex by interacting with various proteins through its different motifs. FAM21 links the WASH complex to endosomes via retromer binding and inhibits actincapping activity by binding the CAPZ heterodimer via its
C-terminus; the FAM21 N-terminus is required for binding within the WASH complex to maintain its stability ${ }^{17,22-24}$. It is thus important to determine the region of FAM21 responsible for binding SNX27. We found that SNX27 binds two distinct regions within the N-terminal domain of FAM21 (Fig. 1c). Using additional approaches including co-immunoprecipitation (Fig. 1d), glutathione $S$-transferase (GST)-pulldowns (Fig. 1e) and peptide-binding assays (Fig. 1f), we determined that residues $40-79$ and 592-600 of FAM21 are sufficient for binding SNX27 directly.

Binding of FAM21 with SNX27 mediates cargo recycling. The WASH complex has been suggested to be involved in endosometo-plasma membrane transport mediated by the SNX27-retromer complex $^{6,11}$. To investigate whether FAM21 functions in recycling of SNX27-retromer cargoes, we examined the localization of GLUT1, a known cargo of the SNX27-retromer complex, after small interfering RNA (siRNA) transfection. As previously reported ${ }^{6}$, depletion of SNX27 resulted in GLUT1 accumulation at LAMP1-positive lysosomes (Fig. 2a,b). Intriguingly, GLUT1 accumulated in the perinuclear region, not at lysosomes, in FAM21-depleted cells (Fig. 2a,b). Immunofluorescence staining of GM130, a cis-Golgi matrix protein, revealed that depletion of FAM21 caused a selective accumulation of GLUT1 in the Golgi region (Fig. 2c,d). This finding was observed using two different siRNA sequences targeting FAM21 (Fig. 2d and Supplementary Fig. 1), and thus it is likely not an off-target effect.

The mis-sorting of GLUT1 to the endocytic recycling compartment, which is adjacent to the Golgi apparatus, was recently reported ${ }^{25}$. To determine the precise location of missorted GLUT1, we carefully analysed the immunofluorescent signals of GLUT1, GM130 and Tf near the Golgi in FAM21depleted cells. The staining pattern of GLUT1 was continuous, like GM130, rather than more punctate, like Tf (Fig. 2e). Also, the co-localization coefficient of GLUT1 with GM130 was significantly higher than it was with $\mathrm{Tf}$ (Fig. 2f). The mis-sorted GLUT1 disappeared after treatment of brefeldin A (BFA), which dissociates Golgi membrane-associated protein ${ }^{26-28}$, indicating that GLUT1 was associated with the Golgi membranes (Fig. 2g). The GLUT1 released from the Golgi membranes by BFA did not localize to Rab11-positive recycling endosomal tubules, which were also induced by BFA treatment ${ }^{29}$ (Fig. 2g). Together, these results suggest that GLUT1 is mis-sorted to the Golgi apparatus in FAM21-depleted cells.

To examine recycling of another SNX27-retromer cargo, we generated hTERT-RPE1 cells stably expressing $\beta 2 \mathrm{AR}$. The $\beta 2 \mathrm{AR}$ also accumulated at the Golgi apparatus in FAM21-depleted cells (Fig. 2h,i). Mis-sorting defects induced by FAM21 depletion seemed specific to SNX27-retromer cargoes because the localization of $\mathrm{Na}, \mathrm{K}-\mathrm{ATPase}$, a transmembrane protein not affected by SNX27 depletion, was unchanged in FAM21-depleted cells (Supplementary Fig. 2). These results indicate that FAM21 is involved in recycling of SNX27-retromer cargoes. However, the fact that GLUT1 is mis-sorted into a different intracellular compartment upon FAM21 depletion, as compared with SNX27 or retromer depletion, implies a distinct role for FAM21 with retromer in the recycling pathway.

Direct binding of SNX27 with VPS26 is critical for protecting cargoes from lysosomal degradation ${ }^{6}$, and we hypothesized that FAM21 might regulate cargo recycling via SNX27 binding. We depleted endogenous SNX27 in hTERT-RPE1 cells stably expressing siRNA-resistant wild-type (WT) SNX27 or various mutants (Fig. 3a,b). The SNX27 PDZ domain is required for binding cargoes and VPS26, whereas the FERM domain is 
a

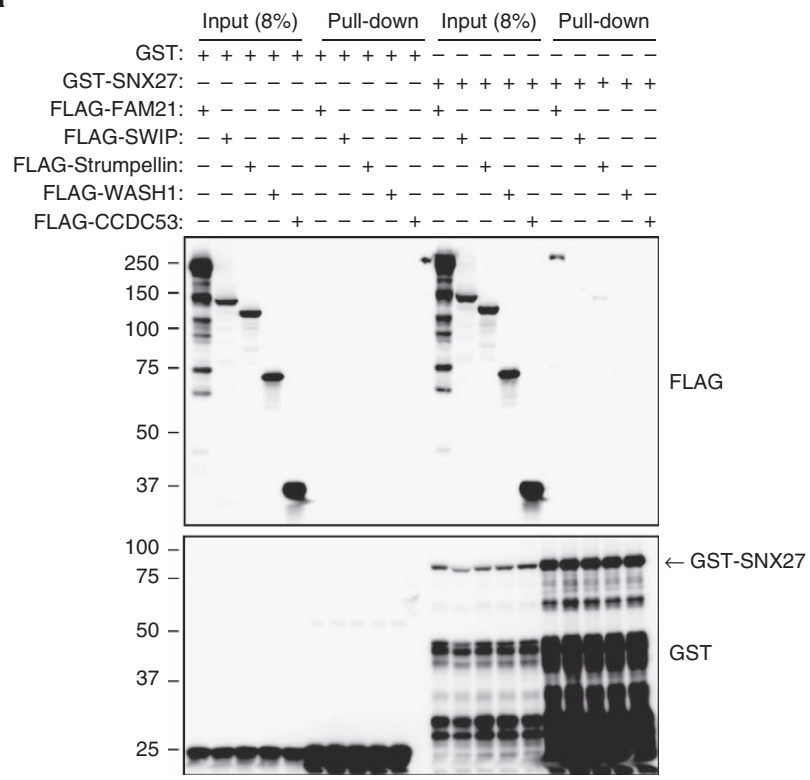

b

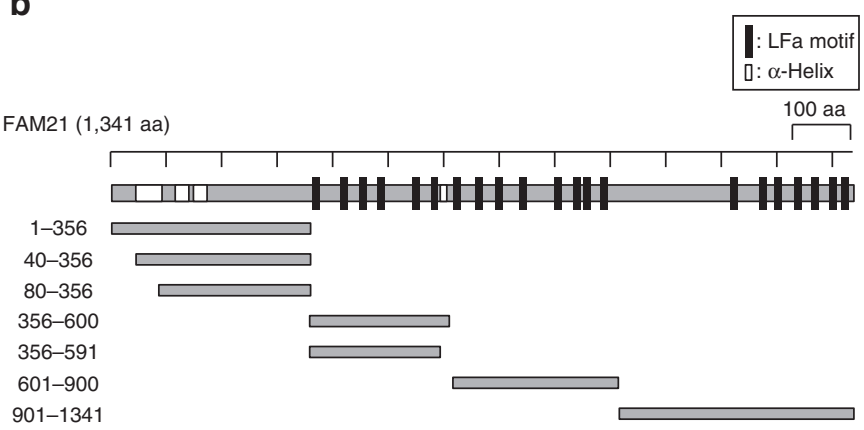

C

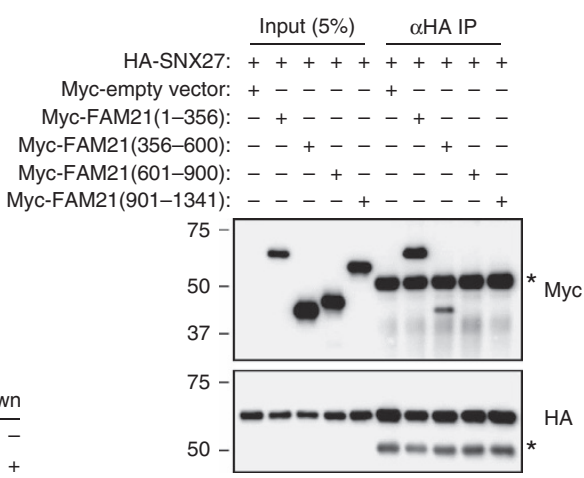

GST-SNX27: $-\cdots++\cdots++$

His-FAM21 (1-600): $+\cdots+-+++$

His-FAM21(80-591): $\quad-+++\cdots+-+$

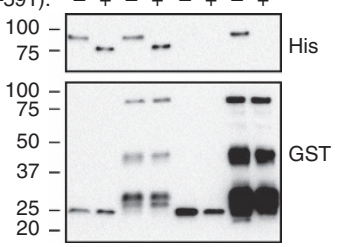

f

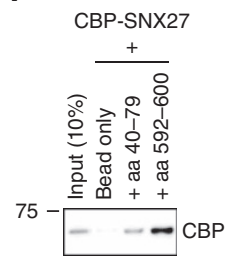

Figure 1 | SNX27 interacts directly with FAM21 among the WASH complex components. (a) HEK293T cells were transfected with $3 \times$ FLAG-taggedWASH complex proteins. Lysates were incubated with GST or GST-SNX27 proteins and precipitated with Glutathione Sepharose 4B beads. Precipitates were then immunoblotted as shown. (b) Schematic representation of FAM21 constructs used. LFa motifs and $\alpha$-helices are indicated. (c,d) HA-SNX27 was co-transfected with the indicated Myc-tagged constructs in HEK293T cells, and lysates were immunoprecipitated with anti-haemagglutinin (HA) antibodies and immunoblotted. Asterisks $\left(^{*}\right)$ denote IgG heavy chains. (e) GST or GST-SNX27 fusion proteins were incubated with indicated His-tagged FAM21 proteins and precipitated using Glutathione Sepharose 4B beads. Precipitates were immunoblotted as indicated. (f) Biotinylated peptides corresponding to FAM21 residues 40-79 and 592-600 were incubated with CBP-SNX27 and precipitated using streptavidin-conjugated beads. Precipitates were immunoblotted for CBP.

required for binding FAM21 (ref. 6; Fig. 3c). As previously reported $^{6}$, lysosomal mis-sorting of GLUT1 by SNX27 depletion was corrected by expression of WT SNX27, but not by the $\triangle \mathrm{PDZ}$ mutant (Fig. 3d,e and Supplementary Fig. 3a). Intriguingly, expression of the $\triangle F E R M$ mutant deficient in FAM21 binding resulted in the same defect as FAM21 depletion-mis-sorting of GLUT1 into the Golgi-suggesting that the phenotype is due to a failure in FAM21 binding (Fig. 3d,e and Supplementary Fig. 3b). Even so, we cannot completely exclude the possibility that other interactions of this domain, such as with Ras and some cargo proteins, play a role.

Consistent with a previous report ${ }^{6}$, depletion of SNX27 caused a reduction in GLUT1 levels, which was recovered by treatment of bafilomycin $\mathrm{Al}$, an inhibitor of vacuolar type $\mathrm{H}^{+}$-ATPase, indicating that the decrease of GLUT1 was caused by enhanced lysosomal degradation (Fig. 3f). The GLUT1 reduction upon SNX27 depletion was also recovered by expression of WT SNX27, but only partially recovered by the $\triangle$ FERM mutant (Fig. 3f), indicating that GLUT1 is not recycled properly and that its lysosomal degradation is still enhanced in the $\triangle \mathrm{FERM}$ mutant cells. Taken together, these data demonstrate that FAM21 regulates proper recycling of SNX27-retromer cargoes via its interaction with SNX27.

FAM21 specifies SNX27 localization at endosomal subdomains. We investigated how FAM21 regulates recycling of SNX27retromer cargoes. First, we examined whether FAM21 depletion affects the localization of SNX27, which serves as a cargo adaptor in the complex. Compared with control cells, the area staining for SNX27 was significantly increased and more concentrated near the Golgi in FAM21-depleted cells (Fig. 4a,b and Supplementary Fig. 4c,d). However, depletion of FAM21 did not alter the puncta size of EEA1, which is associated with the cytoplasmic face of the endosome membrane ${ }^{30}$, indicating that the increase of SNX27 signals is not due to an enlargement of the endosome itself (Supplementary Fig. $4 \mathrm{a}-\mathrm{c}$ ). EEA1 signals were also concentrated near the Golgi in FAM21-depleted cells (Supplementary Fig. 4d), 
a

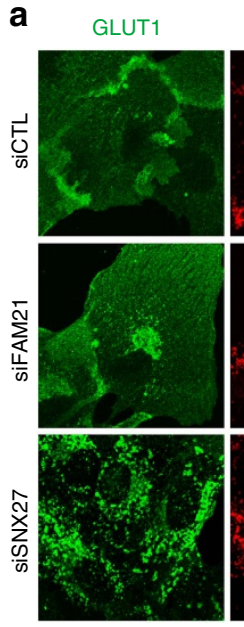

c
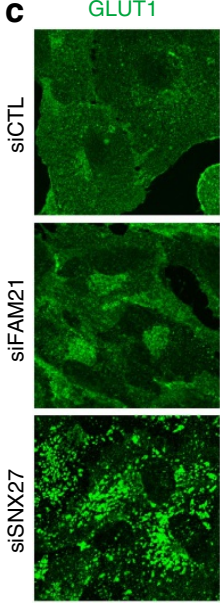

h

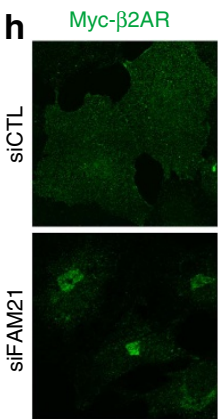

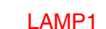

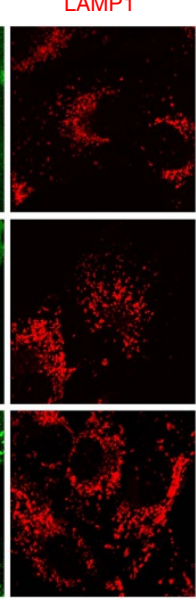

GM130
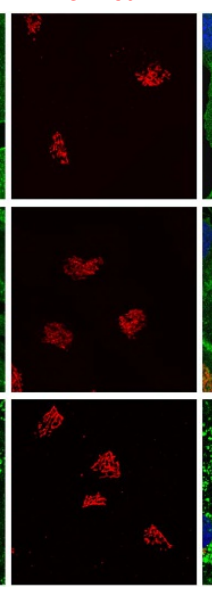

GM130

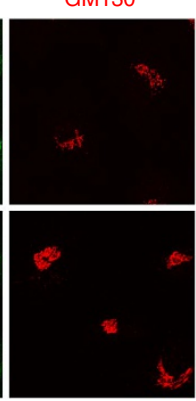

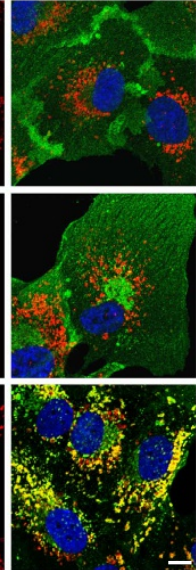

Merge
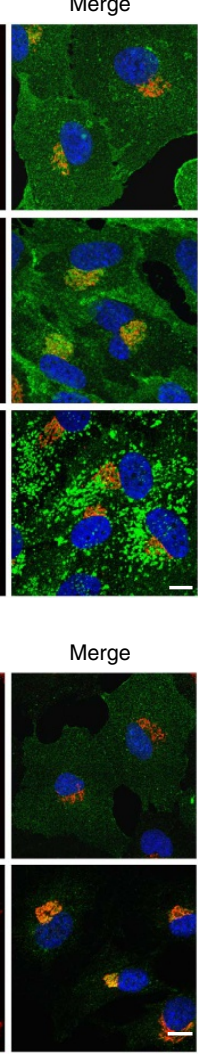

b

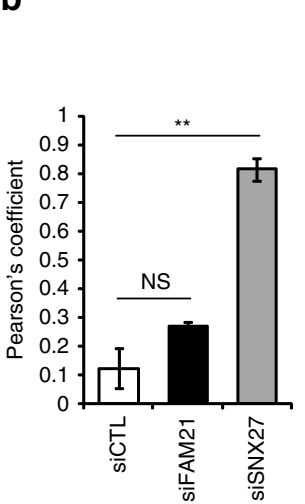

d
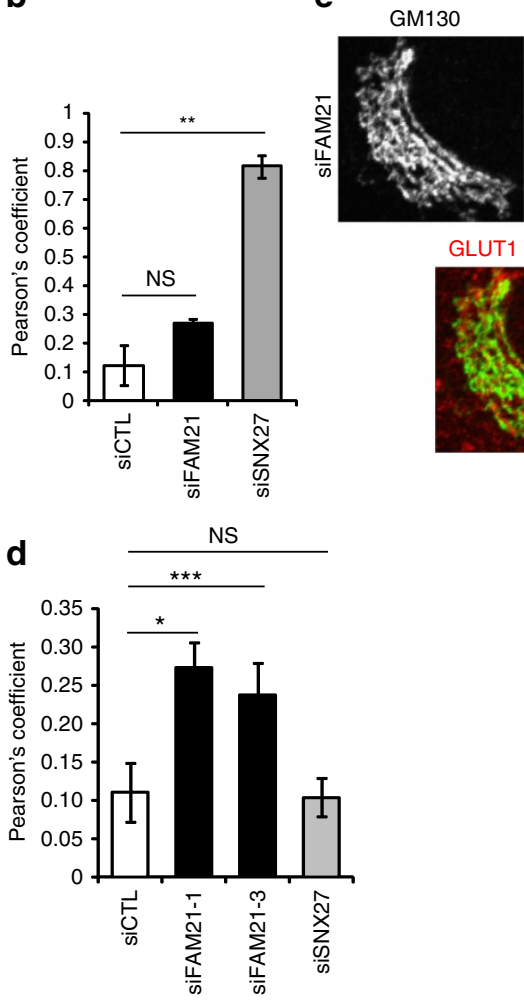

GLUT1

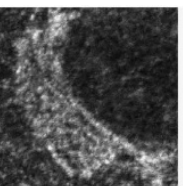

Tf

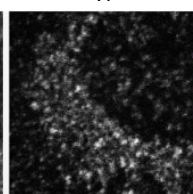

GLUT1 GM130 Tf
GLUT1 Tf

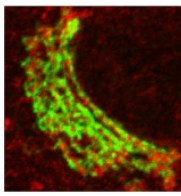

GM130 Tf
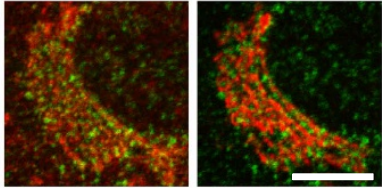

g
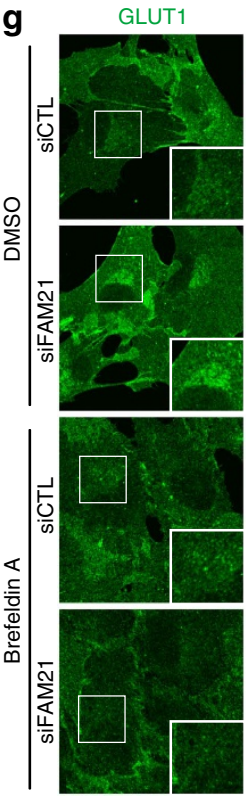

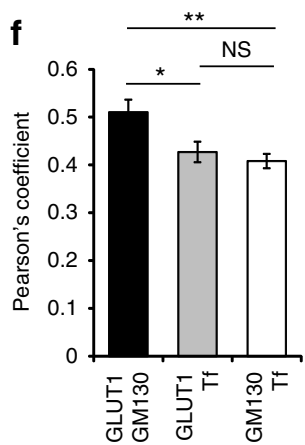

GM130

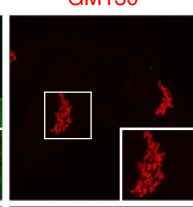

Rab-1
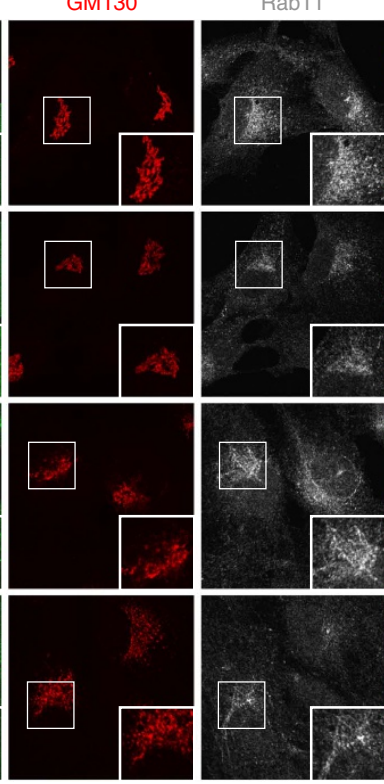

Merge
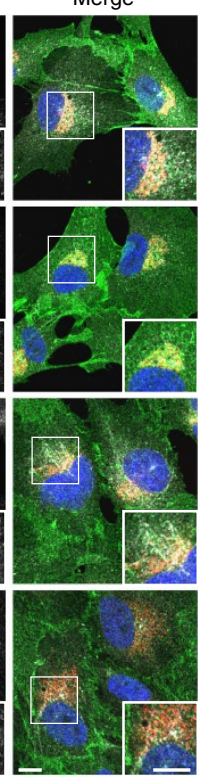

Figure 2 | Depletion of FAM21 causes mis-sorting of SNX27-retromer cargoes to the Golgi apparatus. (a-d) hTERT-RPE1 cells transfected with the indicated specific or control (siCTL) siRNAs were immunostained for GLUT1 (green) along with LAMP1 (a, red) or GM130 (c, red). Co-localization of GLUT1 with LAMP1 (b) or GM130 (d) was analysed by calculation of Pearson's coefficient. Graphs express means \pm s.d. ( $n=3$; $>30$ cells per group). (e) hTERT-RPE1 cells transfected with an siRNA targeting FAM21 were incubated with $10 \mu \mathrm{gl}^{-1}$ of Alexa 568 -conjugated Tf for 20 min at $37^{\circ} \mathrm{C}$ before fixation. Cells were then immunostained for GM130 and GLUT1. For comparison, representative magnified views of the Golgi were chosen and displayed. (f) Co-localization at the Golgi area among GLUT1, GM130 and Tf were analysed by calculation of Pearson's coefficient. Graphs express means \pm s.d. (30 cells per group). (g) hTERT-RPE1 cells transfected with the indicated siRNAs were incubated with $0.05 \%$ of dimethylsulphoxide or $5 \mu \mathrm{g} \mathrm{ml}{ }^{-1}$ of Brefeldin A for 5 min at $37^{\circ} \mathrm{C}$ before fixation and immunostained for GLUT1 (green), GM130 (red) and Rab11 (grey). Insets are magnified views of the Golgi. (h) hTERTRPE1 cells stably expressing Myc- $32 A R$ were transfected with the indicated siRNAs and immunostained for Myc epitope (green) and GM130 (red). (i) Cells with Myc signals accumulating at the Golgi from $\mathbf{h}$ were counted ( $n=2 ; 200$ cells per group). Merged images with 4',6-diamidino-2-phenylindole staining (blue) are to the right. Scale bars, $10 \mu \mathrm{m} .{ }^{\star} P<0.05,{ }^{\star \star} P<0.01,{ }^{\star \star \star} P<0.001 ; \mathrm{NS}$, not significant. 
a

SNX27 (528 aa)

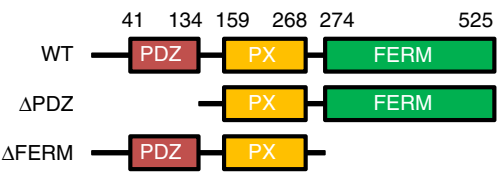

d

d

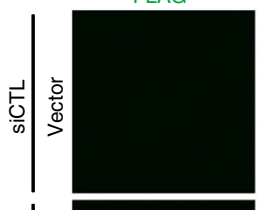

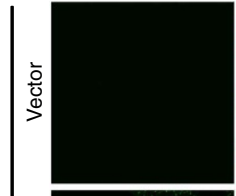

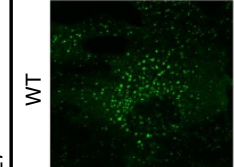

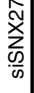

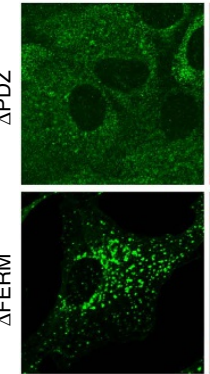

GLUT1

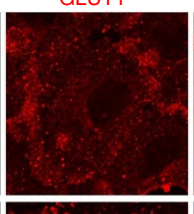

Merge
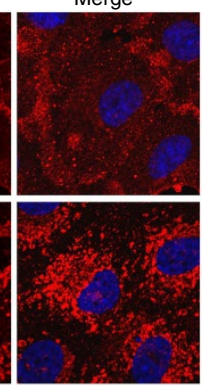

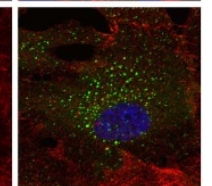

$\mathbf{e}$
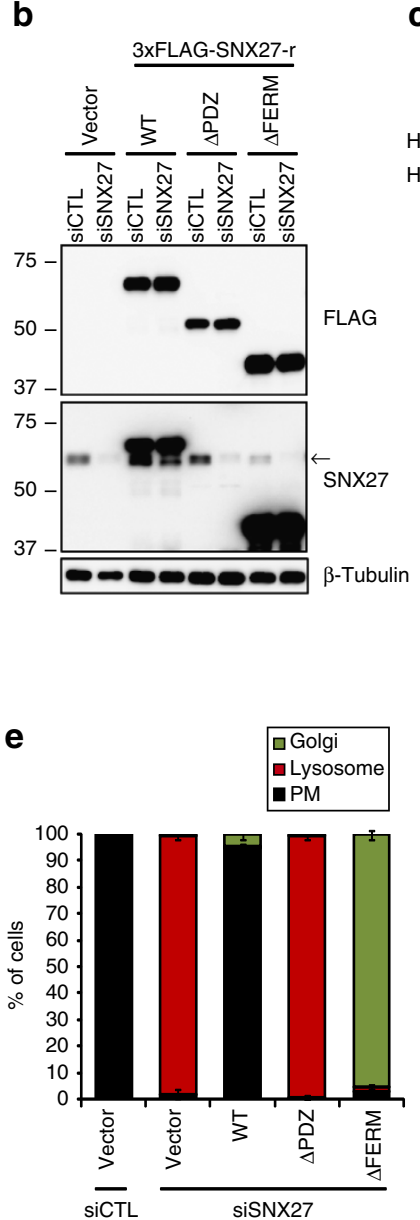

C

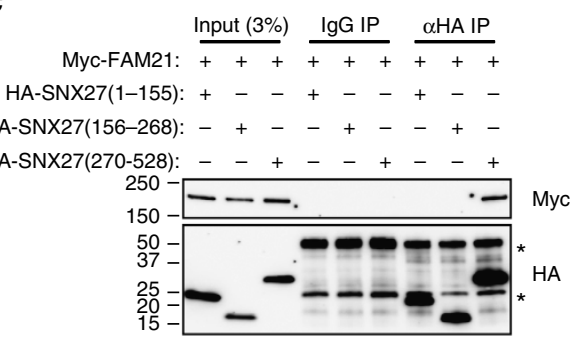

f

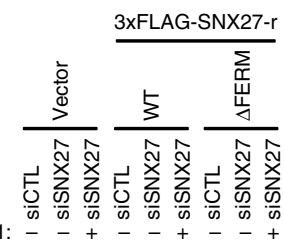

Bafilomycin A1: - - +
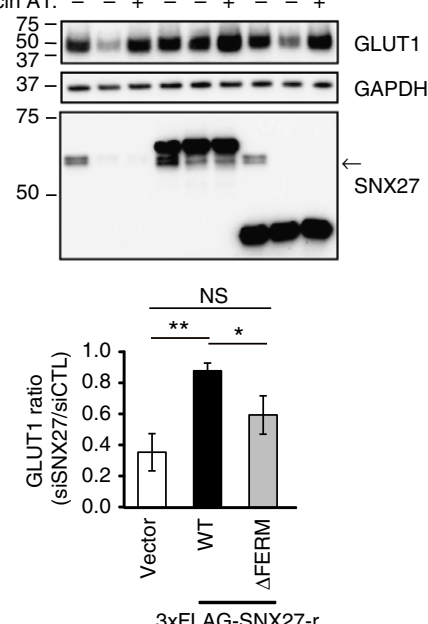

Figure 3 | The interaction of SNX27 with FAM21 is required for proper recycling of its cargo to the plasma membrane (PM). (a) Schematic representation of SNX27 constructs used. (b) hTERT-RPE1 cells stably expressing empty vector or $3 \times$ FLAG-tagged, siRNA-resistant SNX27 constructs were transfected with indicated SNX27 or control (siCTL) siRNAs, and analysed by immunoblotting. An arrow denotes endogenous SNX27. The SNX27 $\triangle \mathrm{PDZ}$ proteins were not detected by anti-SNX27 antibodies because of a deficiency of epitope. (c) Myc-FAM21 was co-transfected with the indicated HASNX27 constructs in HEK293T cells, and lysates were immunoprecipitated with control mouse IgG or anti-haemagglutinin (HA) antibodies. Then, precipitates were immunoblotted as indicated. Asterisks $\left(^{\star}\right)$ denote lgG heavy or light chains. (d) hTERT-RPE1 cells stably expressing empty vector or $3 \times$ FLAG-tagged, siRNA-resistant SNX27 constructs were transfected with the indicated siRNAs and immunostained for FLAG epitope (green) and GLUT1 (red). Merged images with 4',6-diamidino-2-phenylindole staining (blue) are to the right. Scale bar, $10 \mu \mathrm{m}$. (e) Cells were counted based on co-localization of GLUT1 with GM130 (Golgi) or LAMP1 (lysosome) as shown in Supplementary Fig. 3. Cells without accumulated GLUT1 signals were considered as plasma membrane (PM). Graphs express means \pm s.d. ( $n=3 ; 200$ cells per group). (f) hTERT-RPE1 cells stably expressing empty vector or $3 \times$ FLAGtagged, siRNA-resistant SNX27 constructs were transfected with the indicated siRNAs. Twenty-four hours later, cells were cultured in the absence or presence of $50 \mathrm{nM}$ bafilomycin A1 for $24 \mathrm{~h}$, and then analysed by immunoblotting. The intensities of each GLUT1 were normalized with respective GAPDH signals. Levels of GLUT1 in siSNX27-transfected cells relative to siCTL-transfected cells were measured from five independent experiments. Graphs express means \pm s.d. ${ }^{\star} P<0.05,{ }^{\star} * P<0.01 ; N S$, not significant.

indicating that FAM21 loss leads endosomes to be concentrated in the vicinity of the Golgi. FAM21 depletion did not affect total protein levels of the endosomal marker proteins EEA1 and Rab5, but remarkably increased SNX27 levels, as assessed by immunoblotting (Fig. 4c). A recently uncovered role of FAM21 in modulation of nuclear factor- $\kappa \mathrm{B}$ gene transcription led us to examine SNX27 transcript levels ${ }^{31}$. The mRNA level of SNX27 was not affected by FAM21 depletion, suggesting that the increase of SNX27 protein levels would occur in a translational or post-translational manner (Fig. 4d). Together, these results indicate that depletion of FAM21 specifically affects SNX27 levels without interfering with general endosome morphology.

As interaction with FAM21 is critical for SNX27 function, we hypothesized that the increase in SNX27 might be caused by failure to interact with FAM21. We took advantage of cells stably expressing WT SNX27 or the $\triangle$ FERM mutant that is deficient in FAM21 binding. WT SNX27 and $\triangle$ FERM showed distinct localization patterns within endosomes; WT SNX27 was localized to the subdomain of endosomes stained by EEA1, but exactly co-localized with FAM21 (Fig. 4e,f). In contrast, SNX27 $\triangle$ FERM was distributed throughout almost the entire area of endosomes, resulting in an increase of total puncta size as compared with WT (Fig. 4e,g). Specifically, the $\triangle$ FERM mutant formed larger, ringshaped and tubulated endosomes (Fig. 4e, magnified view, green). FAM21 was mostly located at the subdomain or tubules extruding from endosomes decorated by SNX27 $\triangle F E R M$ (Fig. 4f), consistent with a previous study reporting that actin and WASH complex are specifically located along $\beta 2 \mathrm{AR}$-positive tubules on endosomes ${ }^{32}$. Using another cell line expressing a low level of $\triangle F E R M$, we confirmed that the entire endosomal distribution of 


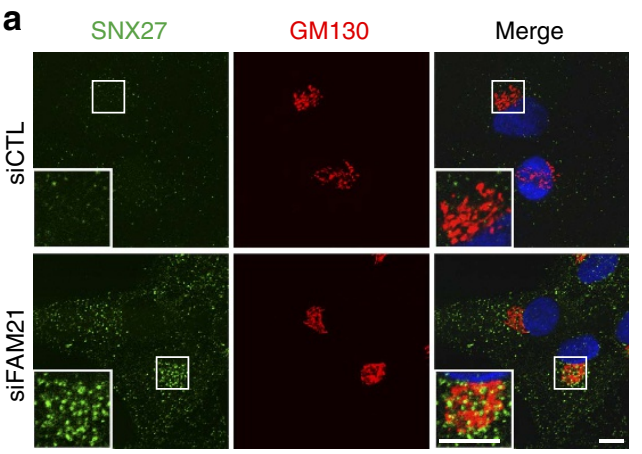

b

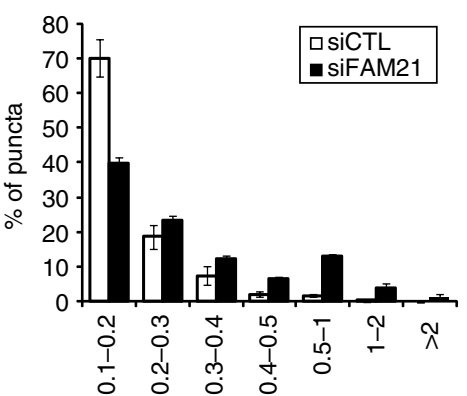

Puncta size decorated by SNX27 $\left(\mu \mathrm{m}^{2}\right)$
C

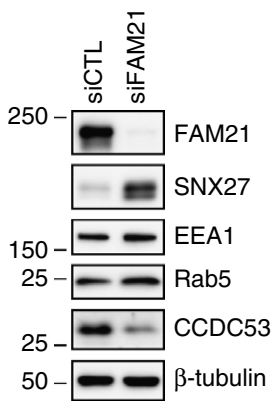

d

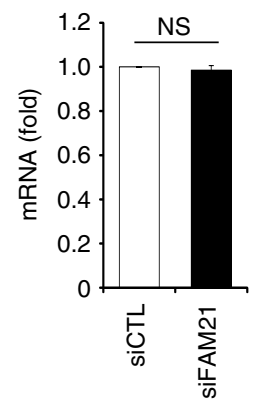

e

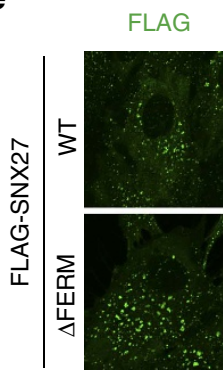

EEA1 Merge
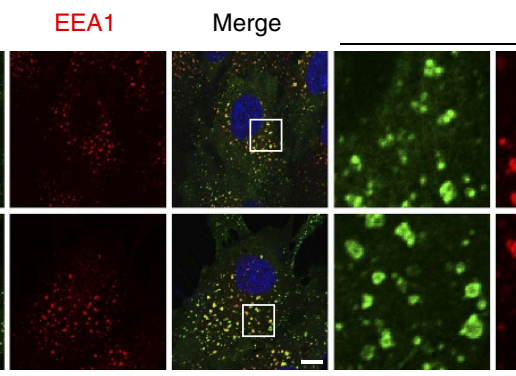

Magnified

f
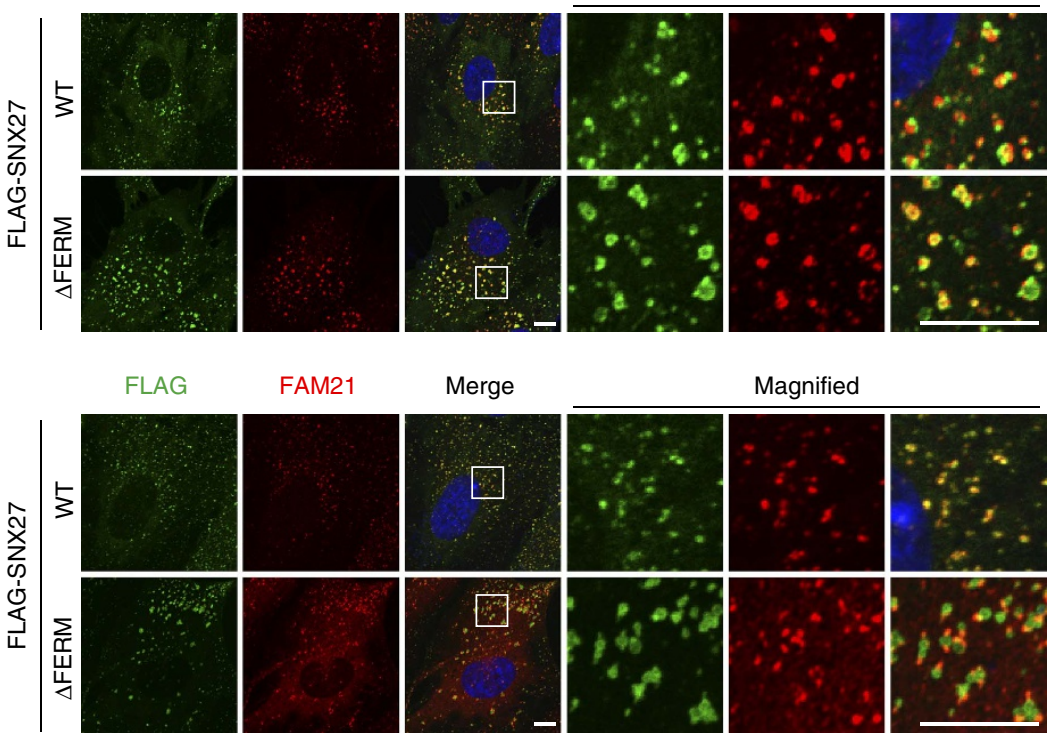

$\mathbf{g}$

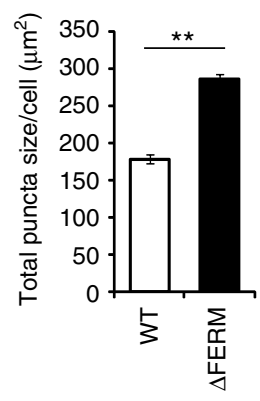

Figure 4 | FAM21 regulates a precise localization of SNX27 at an endosomal subdomain through their interaction. (a) hTERT-RPE1 cells transfected with the indicated SNX27 or control (siCTL) siRNAs were immunostained for SNX27 (green) and GM130 (red). Insets are magnified views of the Golgi area. Merged images with 4',6-diamidino-2-phenylindole staining (blue) are to the right. (b) To measure the size of each punctum, 30 cells of each group were analysed by ImageJ using SNX27 signals from a. (c) hTERT-RPE1 cells transfected with the indicated siRNAs were immunoblotted. (d) The levels of SNX27 mRNA in hTERT-RPE1 cells transfected with the indicated siRNAs were determined by quantitative real-time PCR. (e,f) hTERT-RPE1 cells stably expressing FLAG-SNX27, WT or $\triangle$ FERM mutant were immunostained for FLAG epitope (green) along with EEA1 (e, red) or FAM21 (f, red). Merged images with 4',6-diamidino-2-phenylindole (blue) staining and magnified views of insets are to the right. (g) To measure the total size of puncta visualized by FLAG signals from $\mathbf{e}, 30$ cells of each group were analysed with ImageJ. Graphs express means \pm s.d. ${ }^{\star \star} P<0.01 ;$ NS, not significant. Scale bars, $10 \mu \mathrm{m}$.

$\triangle$ FERM was not due to higher expression than WT (Supplementary Fig. 5). Based on these results, we suggest that SNX27 localizes to a specialized endosomal subdomain via FAM21 interaction to sort cargoes to their correct destination, and that a failure of interaction with FAM21 leads to dispersal of SNX27 throughout the entire surface of endosomes.

FAM21 maintains proper levels of PI(4)P at the Golgi. Given that FAM21 depletion causes accumulation of SNX27 and its cargoes at the Golgi, we examined the effect of FAM21 depletion on the Golgi to gain insight into mechanisms of FAM21 in the recycling pathway. When cells were stained for GM130, a cisGolgi matrix protein, and TGN46, a trans-Golgi membrane protein, the immunostained Golgi region was expanded in FAM21-depleted cells, to about twofold of that in control cells (Fig. 5a,b).

Each intracellular organelle is identified by a specific subset of activated GTPases and specific lipids, permitting in the proper transport of proteins ${ }^{33}$. PI(4)P, a Golgi-enriched phosphoinositide, facilitates release of retromer-associated cargoes at the $\mathrm{TGN}^{34}$. In addition, interactions of the WASH complex with phospholipid and PI(4)P kinase type II $\alpha$ have been reported $^{17,35}$. Therefore, we investigated the relationship of FAM21 with PI(4)P or its kinases. Depletion of FAM21 significantly increased levels of $\mathrm{PI}(4) \mathrm{P}$ and $\mathrm{PI} 4 \mathrm{~KB}$, one of three Golgi-resident PI(4)P kinases, at the Golgi (Fig. 5c-e). The increase of Golgi-localized PI4KB was not accompanied by changes in total PI4KB protein levels, whereas SNX27 protein levels were increased (Fig. 5f).

To examine whether the accumulation of SNX27-retromer cargoes seen in FAM21-depleted cells was caused by an elevation of PI(4)P levels at the Golgi, we depleted PI(4)P in FAM21depleted cells (Fig. 5f). Transfection of an siRNA targeting PI4KB effectively depleted PI(4)P from the Golgi (Supplementary Fig. 6a). Intriguingly, double knockdown of FAM21 and PI4KB did not induce mis-sorting of GLUT1 (Fig. 5g,h), indicating that the mis-sorting is mediated by increased levels of PI(4)P. Similar 
a

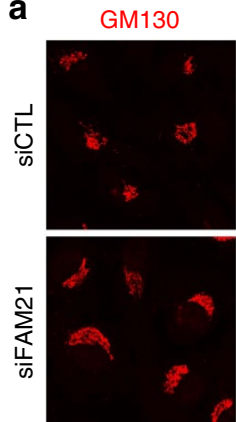

d

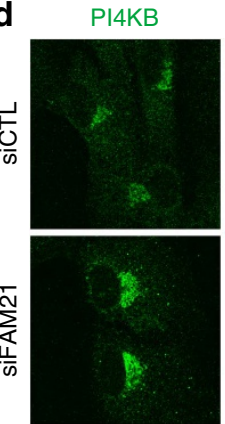

g
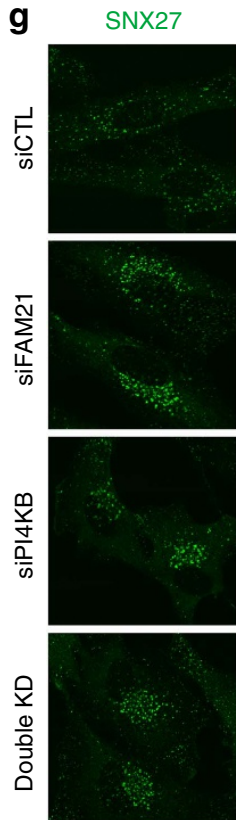

TGN46

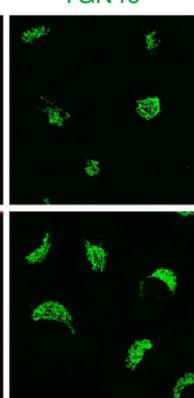

GM130

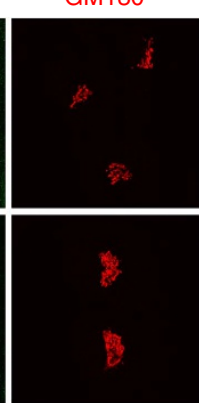

GLUT1
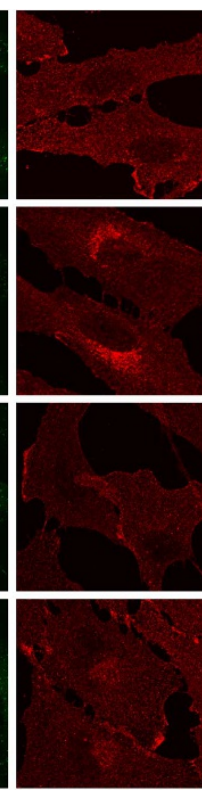

Merge
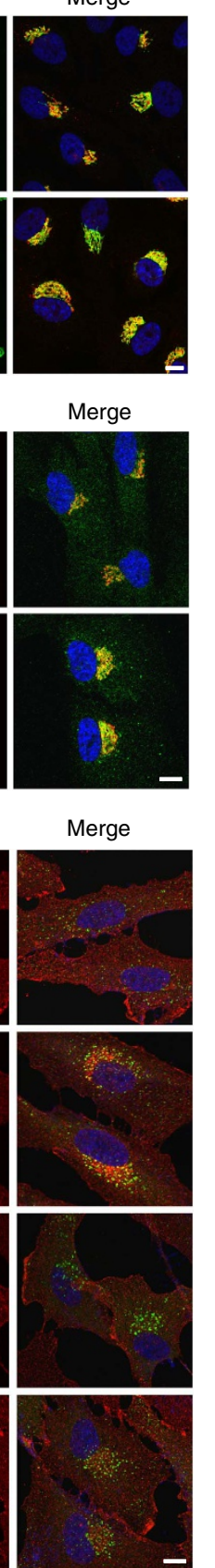

b

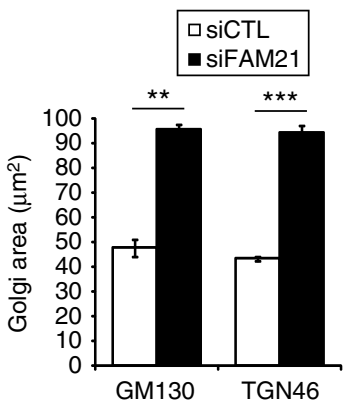

e

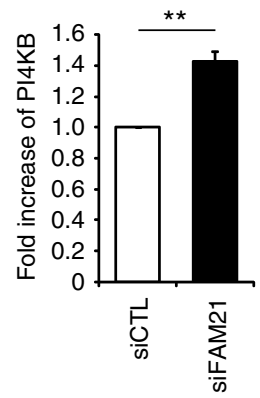

h
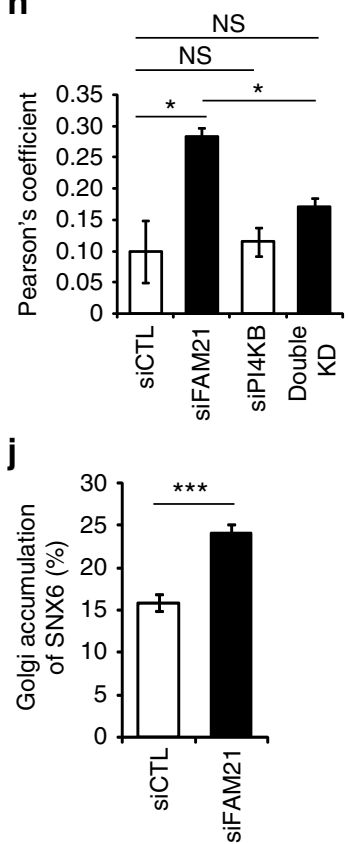

C

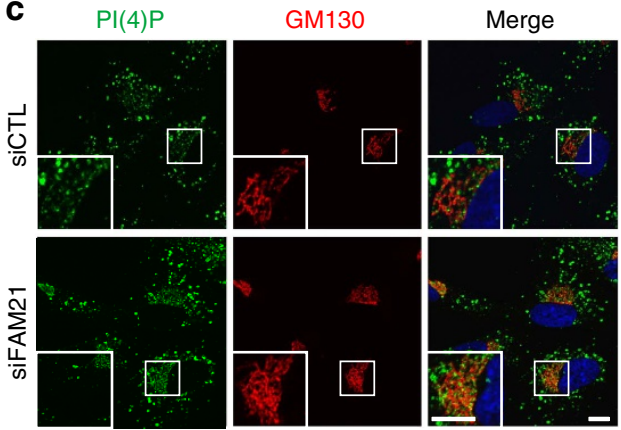

f

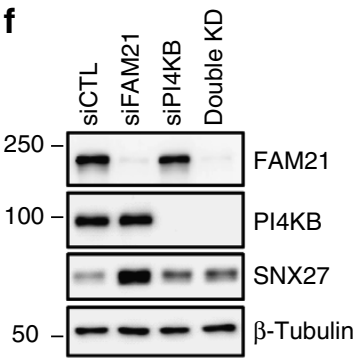

i

SNX6

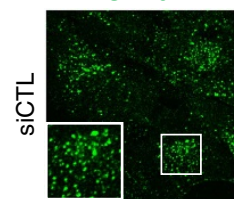

GM130
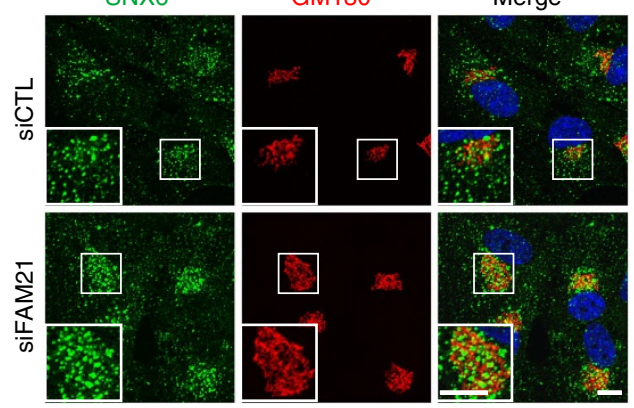

Figure 5 | Mis-sorting of GLUT1 into the Golgi in FAM21-depleted cells is caused by an elevation of PI(4)P levels at the Golgi. (a) hTERT-RPE1 cells transfected with indicated FAM21 or control (siCTL) siRNAs were immunostained for GM130 (red) and TGN46 (green). (b) The area of the Golgi was measured by staining of GM130 or TGN46 from a. Graphs express means \pm s.d. ( $n=3 ; 20$ cells per group). (c,d) hTERT-RPE1 cells transfected with indicated siRNAs were immunostained for GM130 (red) along with PI(4)P (c, green) or PI4KB (d, green). (e) The intensities of PI4KB at the Golgi were measured from 30 cells of three independent experiments from $\mathbf{d}$. Total intensities of PI4KB were divided by the Golgi area. To calculate a fold increase between two groups, averages from FAM21-depleted cells were divided by those of control cells. Graphs express means \pm s.d. ( $n=3 ; 30$ cells per group). (f) hTERT-RPE1 cells transfected with indicated siRNAs were lysed and subjected to immunoblotting. (g) hTERT-RPE1 cells transfected with indicated siRNAs were immunostained for SNX27 (green) and GLUT1 (red). (h) Co-localization between GLUT1 and GM130 was analysed by calculation of Pearson's coefficient. Graphs express means \pm s.d. ( $n=3 ;>30$ cells per group). (i) hTERT-RPE1 cells transfected with indicated siRNAs were immunostained for SNX6 (green) and GM130 (red). (j) The Golgi area was drawn based on GM130 staining, and SNX6 signals in the corresponding area were measured. The area of SNX6 signal per cell was divided by the area of Golgi to calculate the accumulation of SNX6 at the Golgi. Graphs express means \pm s.d. (20 cells per group). Merged images with 4',6-diamidino-2-phenylindole staining are to the right. Insets are magnified views of the Golgi area. Scale bars, $10 \mu \mathrm{m}$. ${ }^{\star} P<0.05,{ }^{\star \star} P<0.01,{ }^{\star \star \star} P<0.001 ; N S$, not significant. 
to the double knockdown result, treatment of FAM21-depleted cells with PAO, a PI(4)-kinase inhibitor, or PIK93, an inhibitor of PI4KB (ref. 36), abolished GLUT1 accumulation at the Golgi (Supplementary Fig. 6b). These data indicate that GLUT1 mis-sorting defects by FAM21 depletion are caused by an elevation of Golgi PI(4)P levels.

Depletion of $\mathrm{PI}(4) \mathrm{P}$ causes an accumulation of retromerassociated vesicles in the cytoplasm ${ }^{34}$. We also observed increased levels of SNX27 in PI4KB-depleted cells by immunostaining (Fig. 5g). However, this increase in PI4KB-depleted cells was not accompanied by increased total protein levels of SNX27, distinct from what we observed in FAM21-depleted cells (Fig. 5f). The increase of SNX27 protein level was suppressed by double knockdown (Fig. 5f), suggesting that the increase in SNX27 is also caused by increased levels of PI(4)P.

$\mathrm{PI}(4) \mathrm{P}$ facilitates dissociation of retromer and its cargoes from the motor at the Golgi by inhibiting the interaction between the p150 Glued of the dynein-dynactin complex and SNX6, a cargo adaptor of retromer ${ }^{34}$. Indeed, we observed an enrichment of SNX6 at the Golgi in FAM21-depleted cells, suggesting that the release of the SNX27-retromer cargoes at the Golgi is mediated by dissociation of SNX6 from the motor via increased PI(4)P levels (Fig. 5i,j).

Distinct function of FAM21 from other WASH components. As FAM21 is a core component of the WASH complex, we examined whether other WASH complex proteins also function in recycling of SNX27-retromer cargoes. However, cells depleted of the WASH components strumpellin or WASH1 did not accumulate GLUT1 and PI4KB at the Golgi (Fig. 6a-d). The possibility that WASH complex members can function at different steps in the exocytosis pathway was recently raised in a study using Dictyostelium ${ }^{37}$. In particular, this study demonstrated that all WASH complex members except FAM21 function to drive actin assembly on lysosomes, whereas FAM21 functions to drive WASH recycling back to lysosomes. Thus, we sought to investigate the distinct phenotypes induced by depletion of FAM21, strumpellin or WASH1 in detail.

Whereas WASH1 and CCDC53 levels were decreased by all three knockdowns, as already demonstrated ${ }^{17}$, levels of FAM21 and strumpellin were not significantly altered by WASH1 depletion (Fig. 6e), and a small amount of FAM21 remained in strumpellin-depleted cells (Fig. 6e). After strumpellin or WASH1 depletion, residual FAM21 was still visible at endosomes, although the FAM21-positive puncta were larger and fewer than in control cells (Fig. 6f). These results demonstrate that FAM21 still localizes to endosomes even in the absence of strumpellin or WASH1, suggesting that the residual FAM21 might be functional in cargo recycling in these cells.

Next, we examined whether SNX27 is affected by depletion of other WASH complex proteins. As in the case of FAM21 depletion, endosomal SNX27 levels were also increased upon depletion of strumpellin or WASH1, with an increase in total protein levels (Fig. 6e,g). Thus, an increase in SNX27 does not always result in GLUT1 and PI4KB accumulation at the Golgi. There are two possible explanations for the above phenotypic differences. The first is that only FAM21 functions in recycling. If so, the increase of SNX27 protein in WASH1- or strumpellindepleted cells might be caused by a different mechanism than in FAM21-depleted cells. We found that depletion of strumpellin or WASH1 induced enlargement of endosomes, which were enriched with the other WASH complex proteins and SNX1, a component of the retromer complex (Fig. 6f,h and Supplementary Fig. 7). The enlarged and collapsed endosomes seen in these cells were previously observed in WASH-knockout fibroblasts $^{38}$. In contrast, FAM21 depletion did not increase endosome size, but it caused loss of WASH complex proteins from the endosome (Fig. 6h and Supplementary Figs 4a-c and 7). Thus, we cannot exclude the possibility that the increase of SNX27 in strumpellin- or WASH1-depleted cells was caused by general expansion of the endosomal network. A second possible explanation is that FAM21 and the rest of the WASH complex function together in recycling of SNX27-retromer cargoes. In this scenario, FAM21 might regulate PI4KB independently of the WASH complex, but function in SNX27 regulation in collaboration with the WASH complex. As the WASH complex comprises a stable assembly, we favour the latter model. Altogether, we suggest that FAM21 acts as a regulator of membrane protein recycling in conjunction with the SNX27-retromer complex.

\section{Discussion}

Once internalized, membrane proteins are transported to one of three different intracellular compartments from endosomes to meet the needs of cells (Fig. 7). For SNX27-retromer cargoes, trafficking to lysosomes is blocked by the interaction of SNX27 with retromer ${ }^{6}$. Here we show that trafficking of cargoes to the Golgi, another route, is blocked by the interaction of SNX27 with FAM21. The interaction limits SNX27 to a specific endosomal subdomain, which is required for cargo export to the plasma membrane, its destination. Furthermore, FAM21 controls PI(4)P levels at the Golgi to prevent unscheduled dissociation of cargoes from the complex. Therefore, we suggest that the SNX27-retromer-WASH complex acts as a hub to direct cargoes to the plasma membrane by blocking their transport to lysosomes and the Golgi.

Our results indicate that FAM21 might have a distinct role from other WASH components in recycling SNX27-retromer cargoes. FAM21, strumpellin and SWIP are known to regulate the stability of the WASH complex ${ }^{17,22}$. Here, we have shown that depletion of strumpellin or WASH1 does not abolish other WASH complex proteins from endosomes, whereas depletion of FAM21 abolishes other proteins (Fig. 6f,h and Supplementary Fig. 7). Our data indicate that FAM21 is the most critical determinant of WASH complex stability. Thus, we infer that residual FAM21 remains functional in strumpellin- or WASH1-depleted cells, so that the accumulation of GLUT1 or PI4KB does not occur. On the other hand, the intact WASH complex appears required for localizing SNX27 to a specific endosomal subdomain, as depletion of any WASH complex protein results in an increase of SNX27 at endosomes (Figs 4 and $6 \mathrm{~g}$ ). Even so, we cannot rule out the possibility that the increase of SNX27 seen in strumpellin- or WASH1-depleted cells is caused by expansion of endosomes ${ }^{38}$ (Fig. 6f,h). In sum, we suggest that the WASH complex functions in limiting SNX27 at the endosomal subdomain, and FAM21 regulates levels of PI4KB at the Golgi independent of the WASH complex. Our suggestion is supported by a recent study showing that FAM21 functions at a different step of exocytosis than other WASH complex components $^{37}$. Also, there are different knockdown phenotypes among FAM21 and other WASH components in endosome size, Golgi morphology and nuclear factor- $\kappa \mathrm{B}$ transcriptional activity $^{22,31,38}$ (Figs 5 and 6 and Supplementary Fig. 4). Thus, growing evidence suggests that FAM21 functions beyond the WASH complex.

Unexpectedly, we found that depletion of FAM21 increases total protein levels of SNX27 (Fig. 4c). As its respective mRNA levels were not changed in FAM21-depleted cells, the increase would not occur at a transcriptional level (Fig. 4d). K63-linked ubiquitination of WASH1 by MAGE-L2-TRIM27, an E3 RING 
a

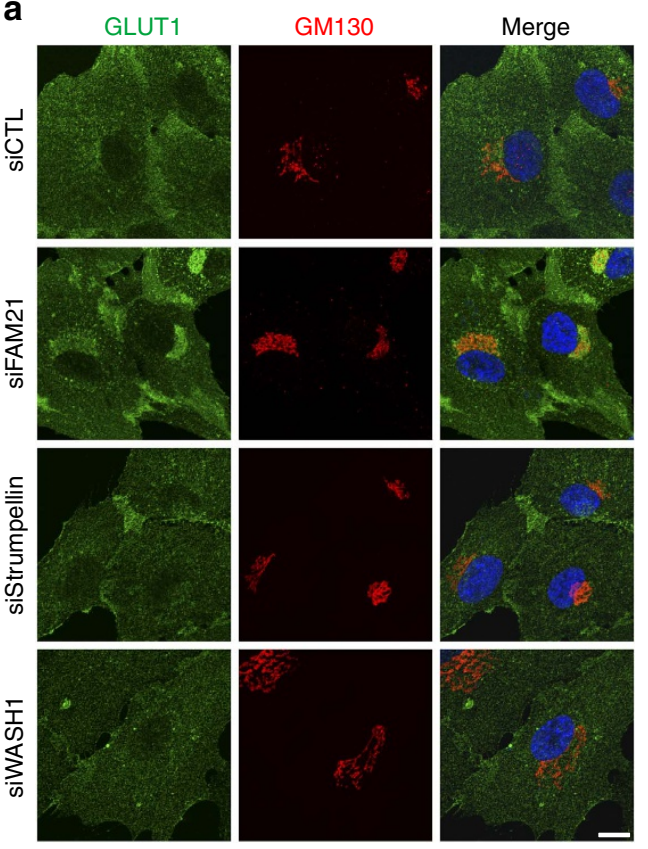

b

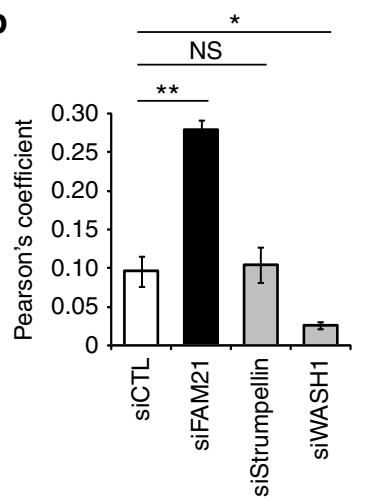

e
C

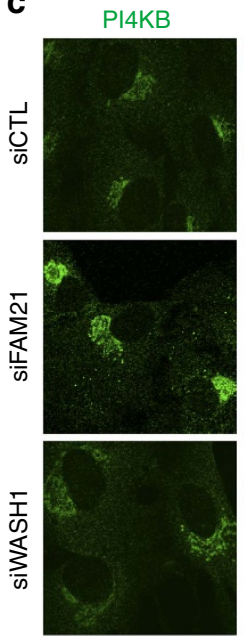

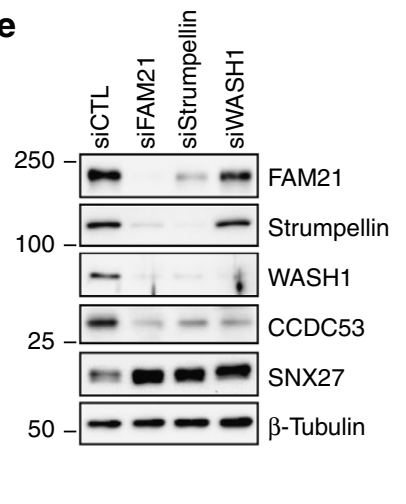

g

SNX27
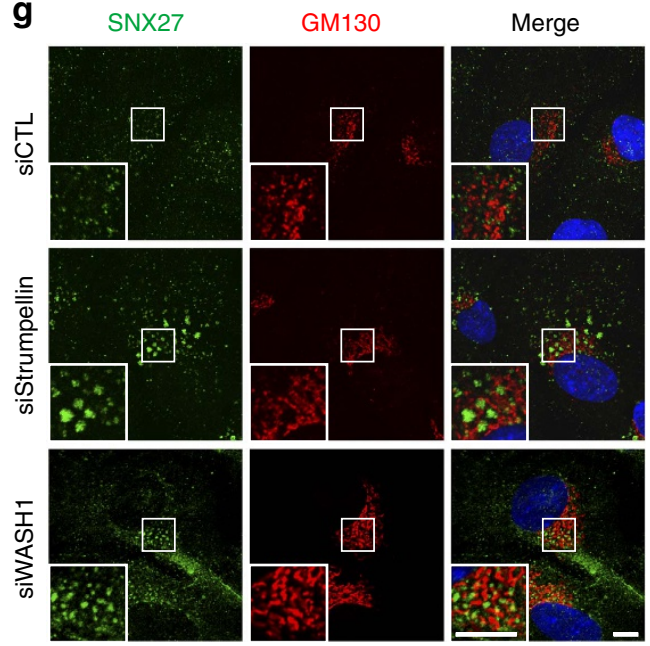

GM130

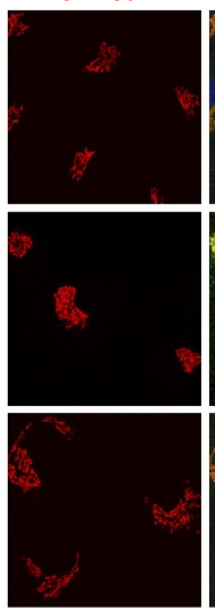

f
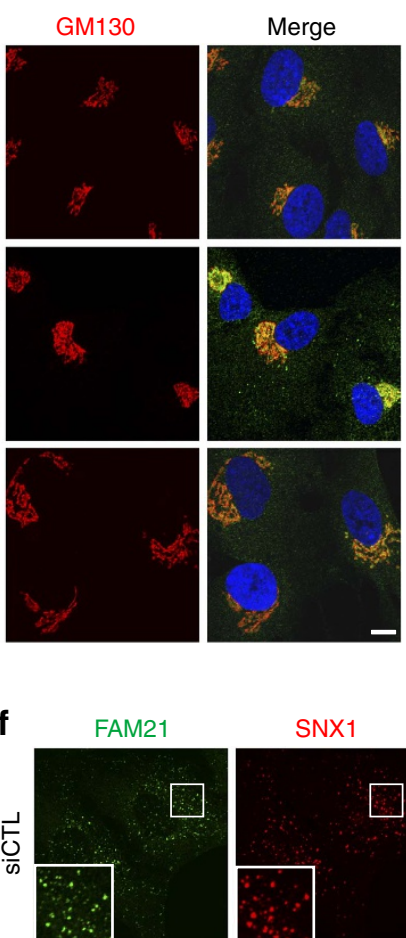

d

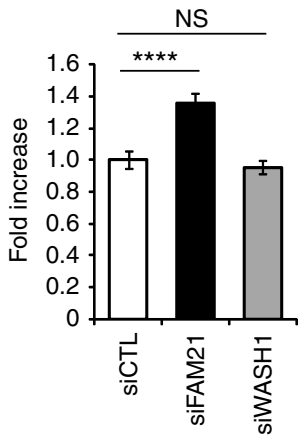

SNX1
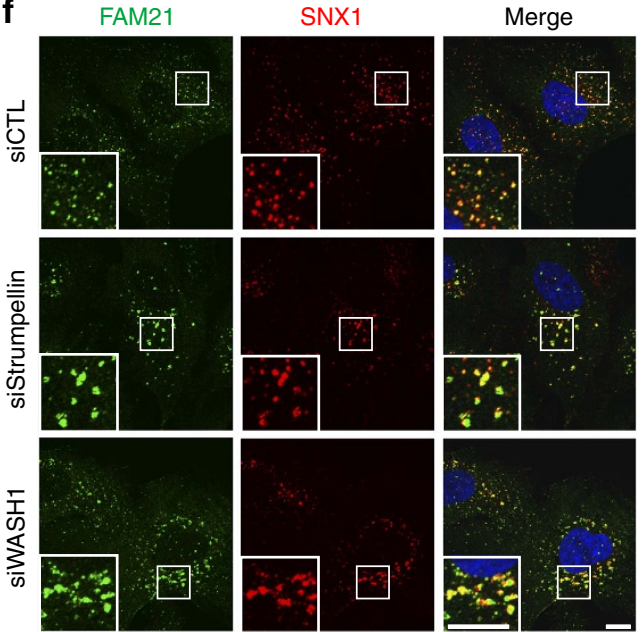

h
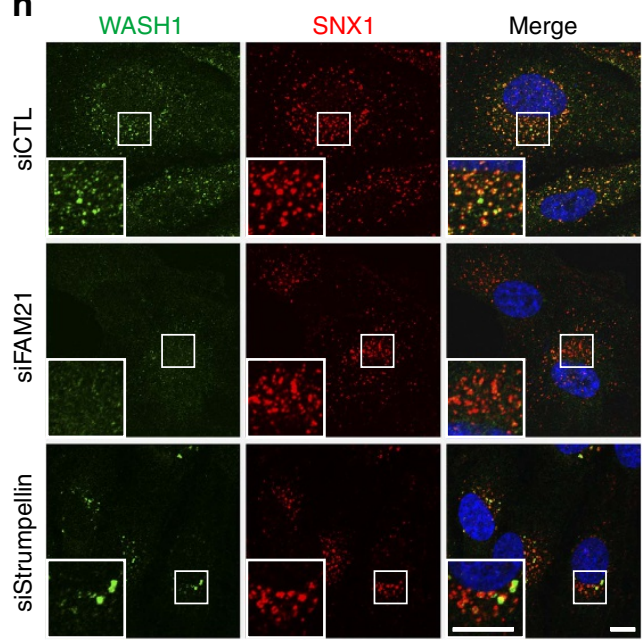

Figure 6 | FAM21 has a distinct function in recycling of SNX27-retromer cargoes from other WASH complex components. (a) hTERT-RPE1 cells transfected with the indicated specific or control (siCTL) siRNAs were immunostained for GLUT1 (green) and GM130 (red). (b) Co-localization between GLUT1 and GM130 was analysed by calculation of Pearson's coefficient. Graphs express means \pm s.d. ( $n=3 ;>30$ cells per group). (c) hTERT-RPE1 cells transfected with indicated siRNAs were immunostained for PI4KB (green) and GM130 (red). (d) Intensities of PI4KB at the Golgi were measured from 30 cells per group from c. Total intensities of PI4KB were divided by the Golgi area. To calculate a fold-increase from control cells, averages of experimental groups were divided by those of control cells. Graphs express means \pm s.d. (e) hTERT-RPE1 cells transfected with the indicated siRNAs were immunoblotted. (f-h) hTERT-RPE1 cells transfected with the indicated siRNAs were immunostained as shown. Insets are magnified views of the Golgi area in g. Merged images with 4',6-diamidino-2phenylindole staining are to the right. Scale bars, $10 \mu \mathrm{m} .{ }^{\star} P<0.05,{ }^{\star \star} P<0.01,{ }^{\star \star \star \star} P<0.0001$; NS, not significant. 


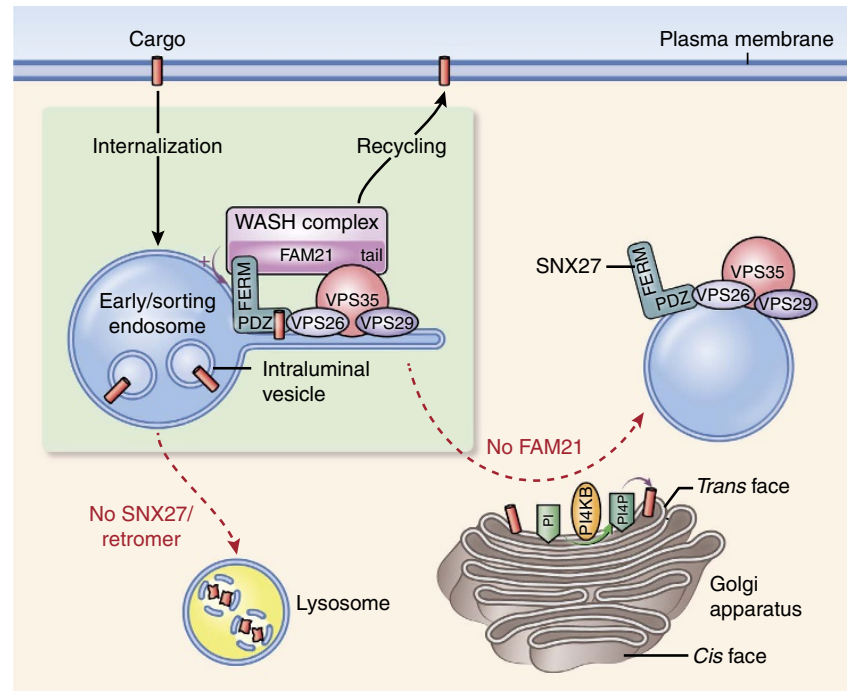

Figure 7 | Model for roles of the SNX27-retromer-WASH complex in cargo sorting. After internalization, cargoes harbouring a PDZ-binding motif such as $\beta 2 A R$ and GLUT1 bind to a PDZ domain of SNX27. SNX27 serves as an adaptor linking its cargoes to the endosomal tubules through its interaction. Interaction of its PDZ domain with VPS26 of retromer prevents lysosomal entry of cargoes, whereas the interaction of its FERM domain with FAM21 of the WASH complex prevents its transport to the Golgi apparatus. Therefore, these interactions within the SNX27-retromerWASH complex direct the cargoes towards recycling to the plasma membrane. The WASH complex, in particular, functions in localizing SNX27 at a specialized endosomal subdomain. In addition, FAM21 controls PI4KB levels at the Golgi, resulting in maintaining proper levels of PI(4)P at the Golgi, which prevents dissociation of cargoes from the complex at the Golgi.

ubiquitin ligase, is known to regulate endosome-to-Golgi and endosome-to-plasma membrane recycling ${ }^{39}$. This finding suggests that proteins which are involved in the endosome-toplasma membrane recycling pathway could be regulated by ubiquitination. The increase in SNX27 levels was accompanied by increased immunofluorescence intensities at the Golgi (Fig. 4). In the absence of PI4KB, both the increase in SNX27 protein levels and the accumulation of GLUT1, an SNX27 cargo, were suppressed in FAM21-depleted cells (Fig. 5f-h). The results suggest that the increase of protein levels is related to the GLUT1 mis-sorting defect mediated by PI(4)P. As FAM21 is required for limiting SNX27 at the specialized subdomain of endosomes to export its cargoes (Fig. 4), SNX27 would get trapped in endosomes and have prolonged lifetimes in FAM21-depleted cells. We infer that it might result in the increase in SNX27 levels observed in FAM21-depleted cells. However, the mechanism underlying how FAM21 regulates SNX27 and PI4KB should be addressed further.

Mutations in several genes implicated in endosomal sorting pathways are linked to inherited neurological disorders ${ }^{40,41}$. VPS35, a subunit of retromer, is mutated in autosomal dominant, late-onset Parkinson disease ${ }^{42,43}$. Mutations in WASH components strumpellin or SWIP cause severe, pure, autosomal dominant hereditary spastic paraplegia or autosomal recessive intellectual disability, respectively ${ }^{44-47}$. It is believed that neurons are more vulnerable to endosomal trafficking defects as neuronal endosomes need to transport signalling molecules through far longer distances than other cell types ${ }^{48}$. Our finding that the SNX27-retromer-WASH complex mainly conducts the endosomal sorting pathway will likely clarify cellular mechanisms underlying these neurological disorders.

\section{Methods}

DNA constructs. Human SNX27 (GenBank NM 030918), FAM21

(GenBank BC082258), SWIP (GenBank NM_001293640), strumpellin (GenBank NM_014846), WASH1 (GenBank NM_182905) and CCDC53 (GenBank NM_016053) cDNAs were obtained from Open Biosystems. WT and truncated mutants of FAM21 were subcloned into pGW1-Myc vector ${ }^{49}$ WT and truncated mutants of SNX27 were subcloned into pGW1-HA vector or pCDH-CMV-MCS-EF1-Puro-3 $\times$ FLAG lentiviral vector (SBI; the sequence for the $3 \times$ FLAG epitope was inserted into $X b a I / E c o$ RI sites), respectively. siRNAresistant SNX27 constructs were generated by introducing three silent, mismatched nucleotides (G537A;U540C;G543C) against the siSNX27 target sequence. All constructs were confirmed by DNA sequencing. For detailed information, see Supplementary Table 1.

Antibodies. The following antibodies were used in the study: anti-FLAG (M2, mouse, Sigma-Aldrich, 1:1,000), anti-GST (B-14, mouse, Santa Cruz Biotechnology, 1:2,000), anti-haemagglutinin (F-7, mouse, Santa Cruz Biotechnology, 1:1,000), anti-c-Myc epitope (9E10, mouse, Santa Cruz Biotechnology, 1:1,000), anti-c-Myc epitope (polyclonal, goat, Bethyl Laboratories, 1:1,000), anti-His (H-3, mouse, Santa Cruz Biotechnology, 1:500), anti-CBP (W-15, Goat, Santa Cruz Biotechnology, 1:500), anti-GLUT1 (polyclonal, rabbit, Abcam, 1:500), anti-LAMP1 (H4A3, mouse, Abcam, 1:200), anti-GM130 (35, mouse, BD Biosciences, 1:400), anti-GM130 (polyclonal, rabbit, Cell Signaling, 1:400), anti-Rab11 (47, mouse, BD Biosciences, 1:100), anti-SNX27 (1C6, mouse, Abcam, 1:100), anti- $\beta$-tubulin (D66, mouse, Sigma-Aldrich, 1:2,000), anti-glyceraldehyde3-phosphate dehydrogenase (GAPDH; 6C5, mouse, Santa Cruz Biotechnology, 1:2,000), anti-FAM21 (polyclonal, rabbit, EMD Millipore, 1:500), anti-EEA1 (14, mouse, BD Biosciences, 1:200), anti-EEA1 (polyclonal, rabbit, Cell Signaling, 1:200), anti-Rab5 (C8B1, rabbit, Cell Signaling, 1:200), anti-CCDC53 (polyclonal, rabbit, EMD Millipore, 1:200), anti-TGN46 (polyclonal, sheep, AbD Serotec,

1:200), anti-PI(4)P (mouse, Echelon, 1:200), anti-PI4KB (7, mouse, BD Biosciences, 1:200), anti-SNX6 (N-19, goat, Santa Cruz Biotechnology, 1:400), anti-strumpellin (C-14, rabbit, Santa Cruz Biotechnology, 1:200), anti-WASH1 (polyclonal, rabbit, EMD Millipore, 1:400), anti-SNX1 (51, mouse, BD Biosciences, 1:500), anti-Na, K-ATPase (464.6, mouse, Abcam, 1:500) and anti-SWIP (polyclonal, rabbit, EMD Millipore, 1:200).

Protein purification and in vitro pull-down assays. SNX27 cDNA was subcloned into pGEX-6P-1 vector (Amersham) to be fused with GST, and FAM21 cDNA was subcloned into pRSET-A vector (Invitrogen) to be fused with polyhistidine $(6 \times$ His). Plasmids were transformed into E. coli strain BL21 (DE3) pLysS (Agilent). E. coli were grown and induced with $0.2 \mathrm{mM}$ isopropyl- $\beta$-D-thiogalactopyranoside at $30^{\circ} \mathrm{C}$ for $3 \mathrm{~h}$. Bacterial cells were collected and proteins were purified with Glutathione Sepharose 4B beads (GST fusions; GE Healthcare) or Ni-NTA spin kit (polyhistidine fusions; Qiagen), as per the manufacturer's instructions. After binding, beads were washed three times with binding buffer, and boiled with SDS-PAGE sample buffer. For peptide pull-down assays, peptides corresponding to residues 40-79 (DAGLLQFLQEFSQQTISRTHEIKKQVDGLIRETKATDCRL) and 592-600 (TLCLQAQRE) of FAM21 were synthesized and biotinylated at the $\mathrm{N}$-terminus (GenScript). $1 \mu \mathrm{g}$ of peptides and $1 \mu \mathrm{g}$ of bacterially purified CBP-tagged SNX27 proteins were incubated in binding buffer $(50 \mathrm{mM}$ Tris- $\mathrm{HCl}$ ( $\mathrm{pH} \mathrm{7.5),} 150 \mathrm{mM} \mathrm{NaCl}, 0.05 \%$ Triton $\mathrm{X}-100)$ at $4{ }^{\circ} \mathrm{C}$ for $16 \mathrm{~h}$, followed by incubation with Streptavidin Sepharose High Performance (GE Healthcare) at $4{ }^{\circ} \mathrm{C}$ for an additional $1 \mathrm{~h}$. Beads were washed three times with binding buffer, and bound proteins were analysed by immunoblotting.

Cell culture, transfection and RNA interference. HEK293T and hTERT-RPE1 cells (American Type Culture Collection) were cultured in DMEM and DMEM/F-12 (Gibco) supplemented with $10 \% \mathrm{FBS}$ (Gibco) at $37^{\circ} \mathrm{C}$ in a $5 \% \mathrm{CO}_{2}$ humidified incubator, respectively. Cell lines used in the experiments were regularly tested for mycoplasma contamination and treated when appropriate. Cells were transfected with GenJet Plus (SignaGen Laboratories) or AvalancheOmni (EZ Biosystems) for plasmid DNAs, and Lipofectamine RNAiMax (Invitrogen) for siRNAs as per the manufacturer's instructions. The following sequences of siRNAs were used: siFAM21-1 (Ambion): 5' -GAACAAAACCCAA GGCAAA-3'; siFAM21-2 (Ambion): 5'-GAGUGAAGUCUGUGGAUAA- $3^{\prime}$; siFAM21-3 (Ambion): 5'-GGACAGUGCCUUUGAGCAA-3'; siSNX27 (Invitrogen): $5^{\prime}$-GGUGAGAAGUUUGUGGUAUAUAAUG-3'; siPI4KB (Invitrogen): 5'-GCUCCUGAGAGAGAAUUCAUCAAGU-3'; siStrumpellin (Dharmacon): 5'-GGAUGAGUCUGUAACGUUU-3'; siWASH1 (Ambion): 5'-ACU ACUUCUAUGUGCCAGA- $3^{\prime}$. Control siRNAs were obtained from Ambion.

Generation of stable cell lines. hTERT-RPE1 cells stably expressing SNX27 WT or mutants were generated by lentiviral infection ${ }^{50}$. pCMV-dR8.2 dvpr and pCMV-VSV-G (Addgene) were co-transfected into HEK293T cells along with respective pCDH-CMV-MCS-EF1-Puro-based constructs. The next day, lentivirus was harvested from the culture media by centrifugation and frozen at $-80^{\circ} \mathrm{C}$. hTERT-RPE1 cells were infected with the indicated viruses, and 2 days later 
infected cells were incubated with $20 \mu \mathrm{g} \mathrm{ml}{ }^{-1}$ of puromycin for selection for an additional 2 days.

Chemicals. Dimethylsulphoxide (D8418), BFA (B5936), PAO (P3075) and PIK93 (SML0546) were obtained from Sigma-Aldrich. Bafilomycin Al (19-148) was purchased from EMD Millipore.

Immunofluorescence staining and confocal microscopy. hTERT-RPE1 cells seeded on coverslips were fixed with $4 \%$ paraformaldehyde for $15 \mathrm{~min}$. Fixed cells were blocked with $3 \%$ normal donkey serum in PBST (PBS with $0.1 \%$ Triton $\mathrm{X}-100$ ) or PBSS (PBS with $0.05 \%$ saponin) for $15 \mathrm{~min}$. Then, cells were incubated with primary antibodies diluted in blocking solution for $2 \mathrm{~h}$, washed four times with PBST or PBSS, incubated with Alexa Fluor-conjugated secondary antibodies (Molecular Probes) diluted in blocking solution for $30 \mathrm{~min}$, and washed with PBST or PBSS four times. For the Tf uptake assay, cells were incubated with $10 \mu \mathrm{g} \mathrm{ml}^{-1}$ of Alexa 568-conjugated Tf (Molecular Probes) for $20 \mathrm{~min}$ at $37^{\circ} \mathrm{C}$ before fixation. $\mathrm{PI}(4) \mathrm{P}$ staining was performed as described previously ${ }^{51}$. Cells were fixed with $2 \%$ paraformaldehyde for $15 \mathrm{~min}$, washed with PBS containing $50 \mathrm{mM} \mathrm{NH}_{4} \mathrm{Cl}$ and permeabilized with $20 \mu \mathrm{M}$ digitonin in Buffer A ( $20 \mathrm{mM}$ pipes ( $\mathrm{pH} 6.8), 137 \mathrm{mM}$ $\mathrm{NaCl}$ and $2.7 \mathrm{mM} \mathrm{KCl}$ ) for $5 \mathrm{~min}$. After washing with Buffer A, cells were blocked with blocking solution ( $5 \%$ normal donkey serum in Buffer A) with $50 \mathrm{mM} \mathrm{NH}_{4} \mathrm{Cl}$ for $45 \mathrm{~min}$. Then, cells were incubated with primary antibodies diluted in blocking solution for $2 \mathrm{~h}$, washed with Buffer A and incubated with secondary antibodies diluted in blocking solution for $45 \mathrm{~min}$. For nuclear staining, 4',6-diamidino-2phenylindole (Molecular Probes) was applied for $5 \mathrm{~min}$. Cells were mounted on a slide glass using Fluoromount-G (SouthernBiotech). Cells were visualized using a Zeiss LSM710 confocal microscope with a $\times 63,1.4$ numerical aperture Plan-Apochromat lens. Images were acquired and processed using ZEN 2009 (Carl Zeiss) and Image (NIH) software.

\section{Quantification of co-localization and statistical analysis. Co-localization} analysis was performed on the confocal sections showing maximum signals of GLUT1, PI4KB or Na,K-ATPase using ImageJ (NIH). JACoP (Just another co-localization plugin) was used to calculate the Pearson's coefficient from more than 30 cells per group, from three independent experiments ${ }^{52}$. Microsoft Excel 2007 was used to analyse statistical data and to draw graphs. Significance levels for comparisons between groups were determined by Student's $t$-tests, and $P<0.05$ was considered significant. The variance was similar between the groups we statistically compared.

\section{Immunoprecipitation and immunoblotting. Immunoprecipitation and} immunoblotting were performed as described previously ${ }^{53}$. In detail, cells were washed with PBS and then lysed in lysis buffer (50 mM Tris- $\mathrm{HCl}(\mathrm{pH} 8.0), 150 \mathrm{mM}$ $\mathrm{NaCl}, 1 \%$ Triton X-100, 5 mM EGTA (pH 8.0), 1.5 mM EDTA (pH 8.0), Complete Protease Inhibitor Cocktail (Roche)) on ice for $30 \mathrm{~min}$. Lysates were clarified by centrifugation $(14,000 \mathrm{~g})$ for $20 \mathrm{~min}$ at $4^{\circ} \mathrm{C}$, and incubated with antibodies at $4{ }^{\circ} \mathrm{C}$ overnight. Protein A/G PLUS-agarose beads were added for $1 \mathrm{~h}$, and then the beads were washed three times with lysis buffer. Bound proteins were resolved by SDS-PAGE and then electrophoretically transferred to nitrocellulose membranes. Membranes were blocked with 5\% nonfat milk in Tris-buffered saline with $0.05 \%$ Tween-20 (TBST) for $30 \mathrm{~min}$, and incubated with primary antibodies in 3\% BSA in TBST at $4{ }^{\circ} \mathrm{C}$ overnight. Blots were washed three times with TBST, incubated with horseradish peroxidase-conjugated secondary antibodies (Santa Cruz Biotechnology, 1:2,000) for $1 \mathrm{~h}$, and then washed three times with TBST. Immun-Star WesternC (Bio-Rad) or Amersham ECL Prime (GE Healthcare) was used to detect immunoreactive proteins. Images were obtained and processed using a ChemiDoc XRS + System with Image Lab software (Bio-Rad). Uncropped blot images are presented in Supplementary Fig. 8.

Quantitative real-time PCR (qRT-PCR). RNA was extracted using the RNeasy Mini Kit (Qiagen) and cDNAs were obtained using SuperScript III First-Strand Synthesis SuperMix for qRT-PCR system (Invitrogen). qRT-PCR was performed with SYBR Green PCR Master Mix (Invitrogen) using Applied Biosystems StepOnePlus System (Applied Biosystems). Experiments were performed in quadruplicate using three independent cDNAs. The qRT-PCR results were normalized to GAPDH and calculated following the $2^{-\Delta \Delta \mathrm{Ct}}$ method $^{54}$. The following sequences of primers were used: GAPDH-F: $5^{\prime}$-GGTCGGAGTCAACG GATTTGGTCG-3'; GAPDH-R: 5' ${ }^{\prime}$-CCTCCGACGCCTGCTTCACCAC-3'; SNX27-F: 5'-GAGCAGGCGAGAAGGAATTG-3'; SNX27-R: 5'-GCTTAGAACA CAGCTGCCTC- $3^{\prime}$

\section{References}

1. Huotari, J. \& Helenius, A. Endosome maturation. EMBO J. 30, 3481-3500 (2011)

2. Platta, H. W. \& Stenmark, H. Endocytosis and signaling. Curr. Opin. Cell Biol. 23, 393-403 (2011)
3. Bonifacino, J. S. \& Rojas, R. Retrograde transport from endosomes to the trans-Golgi network. Nat. Rev. Mol. Cell Biol. 7, 568-579 (2006).

4. Burd, C. \& Cullen, P. J. Retromer: a master conductor of endosome sorting. Cold Spring Harb. Perspect. Biol. 6, a016774 (2014).

5. Seaman, M. N. J. The retromer complex - endosomal protein recycling and beyond. J. Cell Sci. 125, 4693-4702 (2012).

6. Steinberg, F. et al. A global analysis of SNX27-retromer assembly and cargo specificity reveals a function in glucose and metal ion transport. Nat. Cell Biol. 15, 461-471 (2013).

7. Seaman, M. N. J., McCaffery, J. M. \& Emr, S. D. A membrane coat complex essential for endosome-to-Golgi retrograde transport in yeast. J. Cell Biol. 142, 665-681 (1998).

8. Arighi, C. N. et al. Role of the mammalian retromer in sorting of the cation-independent mannose 6-phosphate receptor. J. Cell Biol. 165, 123-133 (2004).

9. Carlton, J. et al. Sorting nexin-1 mediates tubular endosome-to-TGN transport through coincidence sensing of high- curvature membranes and 3phosphoinositides. Curr. Biol. 14, 1791-1800 (2004).

10. Seaman, M. N. J. Cargo-selective endosomal sorting for retrieval to the Golgi requires retromer. J. Cell Biol. 165, 111-122 (2004).

11. Temkin, P. et al. SNX27 mediates retromer tubule entry and endosome-toplasma membrane trafficking of signalling receptors. Nat. Cell Biol. 13, 715-721 (2011).

12. Bonifacino, J. S. \& Hurley, J. H. Retromer. Curr. Opin. Cell Biol. 20, 427-436 (2008).

13. Wassmer, T. et al. The retromer coat complex coordinates endosomal sorting and dynein-mediated transport, with carrier recognition by the trans-Golgi network. Dev. Cell 17, 110-122 (2009).

14. Harterink, M. et al. A SNX3-dependent retromer pathway mediates retrograde transport of the Wnt sorting receptor Wntless and is required for Wnt secretion. Nat. Cell Biol. 13, 914-923 (2011).

15. Johannes, L. \& Wunder, C. The SNXy flavours of endosomal sorting. Nat. Cell Biol. 13, 884-886 (2011).

16. Cullen, P. J. \& Korswagen, H. C. Sorting nexins provide diversity for retromerdependent trafficking events. Nat. Cell Biol. 14, 29-37 (2012).

17. Jia, D. et al. WASH and WAVE actin regulators of the Wiskott-Aldrich syndrome protein (WASP) family are controlled by analogous structurally related complexes. Proc. Natl Acad. Sci. USA 107, 10442-10447 (2010).

18. Rottner, K., Hänisch, J. \& Campellone, K. G. WASH, WHAMM and JMY: regulation of Arp2/3 complex and beyond. Trends Cell Biol. 20, 650-661 (2010).

19. Rotty, J. D., Wu, C. \& Bear, J. E. New insights into the regulation and cellular functions of the ARP2/3 complex. Nat. Rev. Mol. Cell Biol. 14, 7-12 (2013).

20. Derivery, E. et al. The Arp2/3 activator WASH controls the fission of endosomes through a large multiprotein complex. Dev. Cell 17, 712-723 (2009).

21. Zech, T. et al. The Arp $2 / 3$ activator WASH regulates $\alpha 5 \beta 1$-integrin-mediated invasive migration. J. Cell Sci. 124, 3753-3759 (2011).

22. Gomez, T. S. \& Billadeau, D. D. A FAM21-containing WASH complex regulates retromer-dependent sorting. Dev. Cell 17, 699-711 (2009).

23. Jia, D., Gomez, T. S., Billadeau, D. D. \& Rosen, M. K. Multiple repeat elements within the FAM21 tail link the WASH actin regulatory complex to the retromer. Mol. Biol. Cell 23, 2352-2361 (2012).

24. Hernandez-Valladares, M. et al. Structural characterization of a capping protein interaction motif defines a family of actin filament regulators. Nat. Struct. Mol. Biol. 17, 497-503 (2010).

25. McGough, I. J. et al. Identification of molecular heterogeneity in SNX27retromer-mediated endosome-to-plasma-membrane recycling. J. Cell Sci. 127, 4940-4953 (2014).

26. Donaldson, J. G., Finazzi, D. \& Klausner, R. D. Brefeldin A inhibits Golgi membrane-catalysed exchange of guanine nucleotide onto ARF protein. Nature 360, 350-352 (1992).

27. Godi, A. et al. ARF mediates recruitment of PtdIns-4-OH kinase- $\beta$ and stimulates synthesis of PtdIns(4,5) $\mathrm{P}_{2}$ on the Golgi complex. Nat. Cell Biol. 1, 280-287 (1999).

28. Wang, Y. J. et al. Phosphatidylinositol 4 phosphate regulates targeting of clathrin adaptor AP-1 complexes to the Golgi. Cell 114, 299-310 (2003).

29. Lippincott-Schwartz, J., Yuan, L. \& Michel-Servet, R. Brefeldin A's effects on endosomes, lysosomes, and the TGN suggest a general mechanism for regulating organelle structure and membrane traffic. Cell 67, 601-616 (1991).

30. Mu, F. T. et al. EEA1, an early endosome-associated protein. EEA1 is a conserved alpha- helical peripheral membrane protein flanked by cysteine "fingers" and contains a calmodulin-binding IQ motif. J. Biol. Chem. 270, 13503-13511 (1995).

31. Deng, Z.-H. et al. Nuclear FAM21 participates in NF-кB-dependent gene regulation in pancreatic cancer cells. J. Cell Sci. 128, 373-384 (2015).

32. Puthenveedu, M. A. et al. Sequence-dependent sorting of recycling proteins by actin-stabilized endosomal microdomains. Cell 143, 761-773 (2010). 
33. Behnia, R. \& Munro, S. Organelle identity and the signposts for membrane traffic. Nature 438, 597-604 (2005).

34. Niu, Y. et al. PtdIns(4)P regulates retromer-motor interaction to facilitate dynein-cargo dissociation at the trans-Golgi network. Nat. Cell Biol. 15, 417-429 (2013).

35. Ryder, P. V. et al. The WASH complex, an endosomal Arp2/3 activator, interacts with the Hermansky-Pudlak syndrome complex BLOC-1 and its cargo phosphatidylinositol-4-kinase type II $\alpha$. Mol. Biol. Cell 24, 2269-2284 (2013).

36. Knight, Z. A. et al. A pharmacological map of the PI3-K family defines a role for p110 $\alpha$ in insulin signaling. Cell 125, 733-747 (2006).

37. Park, L. et al. Cyclical action of the WASH complex: FAM21 and capping protein drive WASH recycling, not initial recruitment. Dev. Cell 24, 169-181 (2013).

38. Gomez, T. S., Gorman, J. A., de Narvajas, A. A.-M., Koenig, A. O. \& Billadeau, D. D. Trafficking defects in WASH-knockout fibroblasts originate from collapsed endosomal and lysosomal networks. Mol. Biol. Cell 23, 3215-3228 (2012).

39. Hao, Y.-H. et al. Regulation of WASH-dependent actin polymerization and protein trafficking by ubiquitination. Cell 152, 1051-1064 (2013).

40. Blackstone, C. Cellular pathways of hereditary spastic paraplegia. Annu. Rev. Neurosci. 35, 25-47 (2012).

41. Neefjes, J. \& van der Kant, R. Stuck in traffic: an emerging theme in diseases of the nervous system. Trends Neurosci. 37, 66-76 (2014).

42. Vilariño-Güell, C. et al. VPS35 mutations in Parkinson disease. Am. J. Hum. Genet. 89, 162-167 (2011).

43. Zimprich, A. et al. A mutation in VPS35, encoding a subunit of the retromer complex, causes late-onset Parkinson disease. Am. J. Hum. Genet. 89, 168-175 (2011).

44. Valdmanis, P. N. et al. Mutations in the KIAA0196 gene at the SPG8 locus cause hereditary spastic paraplegia. Am. J. Hum. Genet. 80, 152-161 (2007).

45. de Bot, S. T. et al. Pure adult-onset spastic paraplegia caused by a novel mutation in the KIAA0196 (SPG8) gene. J. Neurol. 260, 1765-1769 (2013).

46. Xianling, W., Yanhui, Y., Xiangbo, W., Cunjiang, L. \& Jianping, J. A novel KIAA0196 (SPG8) mutation in a Chinese family with spastic paraplegia. Chin. Med. J. 127, 1987-1989 (2014).

47. Ropers, F. et al. Identification of a novel candidate gene for non-syndromic autosomal recessive intellectual disability: the WASH complex member SWIP. Hum. Mol. Genet. 20, 2585-2590 (2011).

48. Yap, C. C. \& Winckler, B. Harnessing the power of the endosome to regulate neural development. Neuron 74, 440-451 (2012).

49. Zhu, P.-P. et al. Cellular localization, oligomerization, and membrane association of the hereditary spastic paraplegia 3A (SPG3A) protein atlastin. J. Biol. Chem. 278, 49063-49071 (2003).
50. Lee, S. et al. MITD1 is recruited to midbodies by ESCRT-III and participates in cytokinesis. Mol. Biol. Cell 23, 4347-4361 (2012).

51. Hammond, G. R. V., Schiavo, G. \& Irvine, R. F. Immunocytochemical techniques reveal multiple, distinct cellular pools of PtdIns4P and PtdIns(4,5)P2. Biochem. J. 422, 23-35 (2009).

52. Bolte, S. \& Cordelières, F. P. A guided tour into subcellular colocalization analysis in light microscopy. J. Microsc. 224, 213-232 (2006).

53. Chang, J., Lee, S. \& Blackstone, C. Spastic paraplegia proteins spastizin and spatacsin mediate autophagic lysosome reformation. J. Clin. Invest. 124, 5249-5262 (2014).

54. Schmittgen, T. D. \& Livak, K. J. Analyzing real-time PCR data by the comparative C(T) method. Nat. Protoc. 3, 1101-1108 (2008).

\section{Acknowledgements}

This work was supported by the Intramural Research Program of the National Institute of Neurological Disorders and Stroke, National Institutes of Health. Ethan Tyler (NIH Medical Arts Branch) provided artistic assistance.

\section{Author contributions}

S.L. and J.C. designed, carried out and interpreted the experiments. S.L., J.C. and C.B. wrote the manuscript.

\section{Additional information}

Supplementary Information accompanies this paper at http://www.nature.com/ naturecommunications

Competing financial interests: The authors declare no competing financial interests.

Reprints and permission information is available online at http://npg.nature.com/ reprintsandpermissions/

How to cite this article: Lee, S. et al. FAM21 directs SNX27-retromer cargoes to the plasma membrane by preventing transport to the Golgi apparatus. Nat. Commun. 7:10939 doi: 10.1038/ncomms10939 (2016)

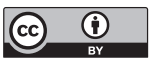

This work is licensed under a Creative Commons Attribution 4.0 International License. The images or other third party material in this article are included in the article's Creative Commons license, unless indicated otherwise in the credit line; if the material is not included under the Creative Commons license, users will need to obtain permission from the license holder to reproduce the material. To view a copy of this license, visit http://creativecommons.org/licenses/by/4.0/ 